
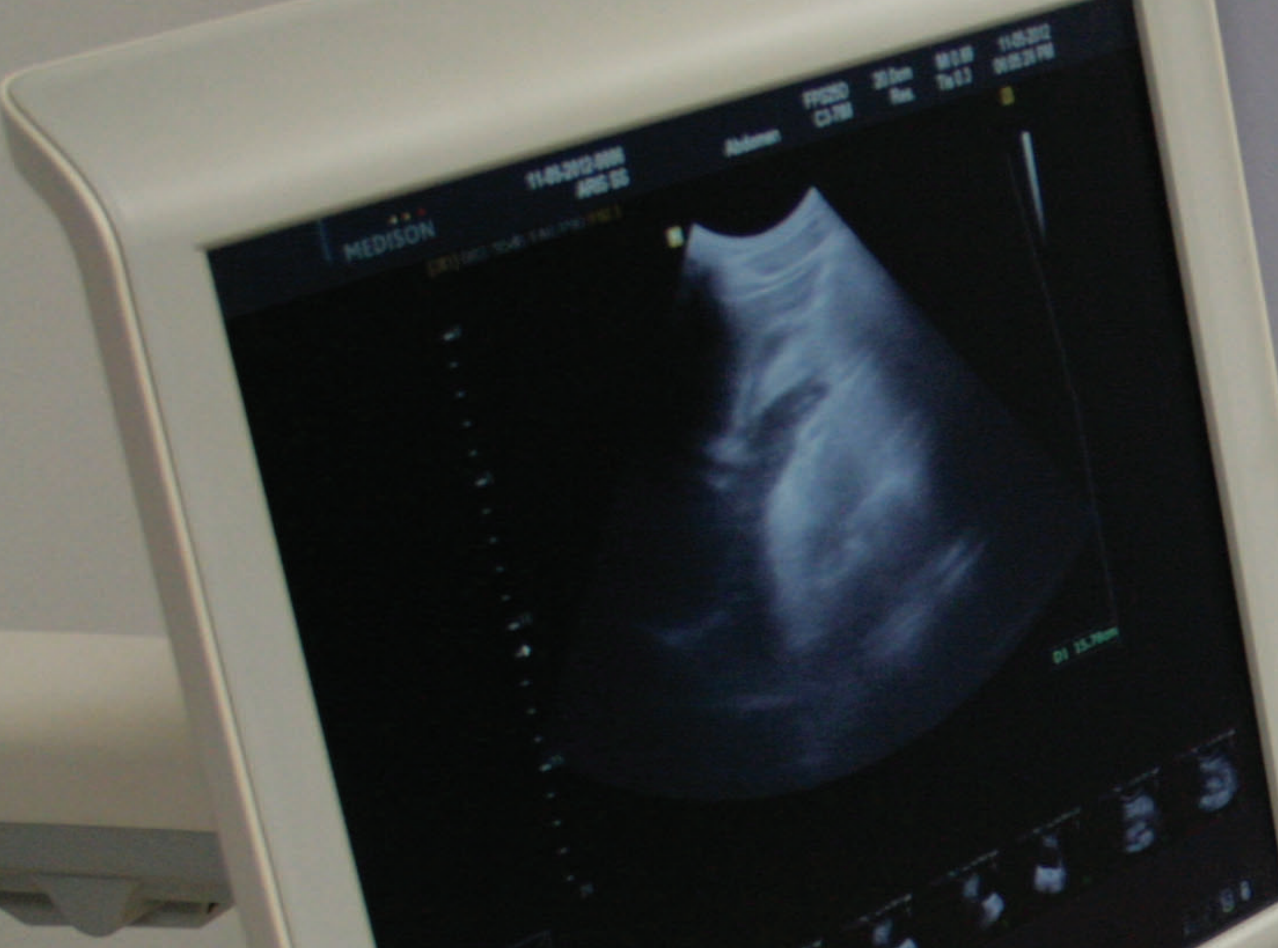

Aims

- To understand the basic principles and practical application of transthoracic ultrasound

- To be familiar with the sonographic appearance of the normal thorax

- To identify basic thoracic pathology

- To utilise ultrasound as guidance for transthoracic interventions

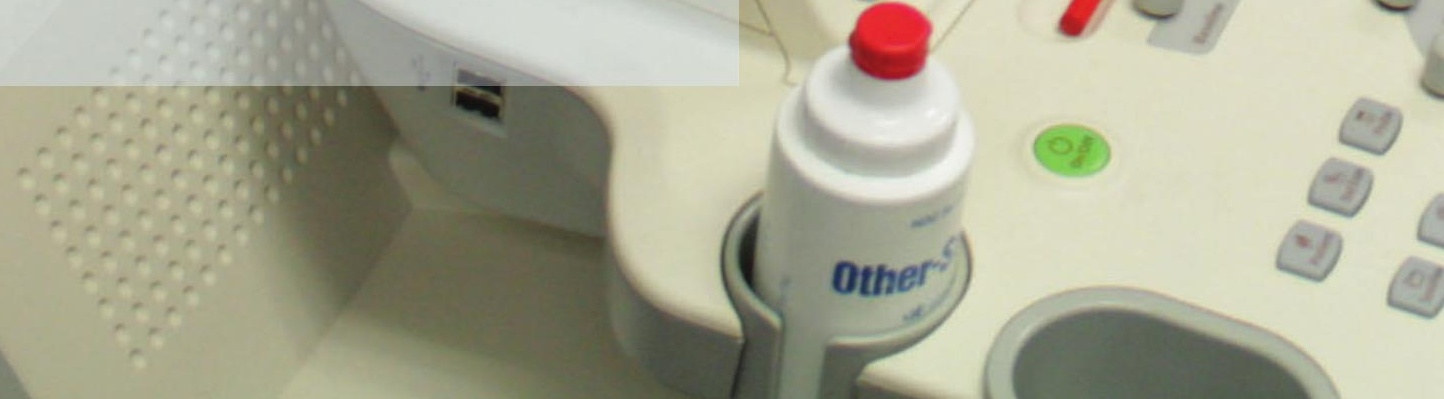




\begin{tabular}{lll}
\hline & $\square$ & D \\
Florian von Groote- & Stellenbosch University & F. von Groote-Bidlingmaier: florianv@sun.ac.za \\
Bidlingmaier, & and Tygerberg Academic & Stellenbosch University, PO \\
Coenraad F.N. & Hospital, Cape Town, & Box 19063, Tygerberg, Cape \\
Koegelenberg & South Africa & Town, 7505, South Africa \\
& &
\end{tabular}

\section{A practical guide to transthoracic ultrasound}

Statement of Interest None declared.

\section{Summary}

Transthoracic ultrasonography is a well-established, yet underutilised imaging modality in respiratory medicine. It allows for real-time and mobile assessment of thoracic disorders and can potentially augment the physical examination of the chest. Moreover, ultrasonography-assisted interventions can be performed by a single clinician without sedation and with minimal monitoring, even outside of the operating theatre. Other advantages of chest ultrasonography include the lack of radiation and the short examination time. Many indications for the use of ultrasonography beyond the visualisation of the pleura and related conditions (including effusions, thickening and pneumothorax) have been validated in the last few decades. These include the assessment of diaphragmatic dysfunction, pulmonary consolidation, interstitial syndromes, pulmonary embolism, and pulmonary and mediastinal tumours, provided they abut the pleura. Transthoracic ultrasonography is an ideal guide for thoracocentesis. Ultrasonography-assisted fine-needle aspiration and/or cutting-needle biopsy of extrathoracic lymph nodes, and lesions arising from the chest wall, pleura, peripheral lung and mediastinum are safe and have a high yield in the of hands of chest physicians. Ultrasonography may also guide aspiration and biopsy of diffuse pulmonary infiltrates, consolidations and lung abscesses, provided the chest wall is abutted. This review will focus on the basic indications and applications for transthoracic ultrasonography.
Transthoracic ultrasonography can be performed with a basic, two-dimensional black-and-white ultrasound system; more sophisticated modalities, like M-mode or colour-flow Doppler, are very rarely indicated. A low-frequency probe (2$5 \mathrm{MHz}$ ) with a curvilinear shape is suitable for screening of superficial and deeper structures, where as a high-frequency probe $(5-10 \mathrm{MHz})$ with a linear shape is used for refined assessment. Higher frequency allows for superior resolution closer to the probe, but at the cost of reduced penetration [1-3]. A clinician should be familiar with the basic controls, including the "freeze", "depth" and "gain" functions [1-3]. The freeze function creates still images, on which measurements can be performed using the track ball. The depth function is a digital zoom that defines what portion of the scanned image is displayed on the monitor at a certain magnification. The scale is displayed on a vertical axis. High-frequency scanning is performed at a maximum depth of around $3-4 \mathrm{~cm}$.
HERMES syllabus link: Module D.3.5 
The depth should preferably be adjusted to allow the area of interest to fill the digital screen. The gain is, in essence, a measure for the amplification of the echoes and determines the brightness of the image, and should be adjusted to maximise the contrast between tissues.

Optimal patient position for scanning is crucial to obtain the best possible images $[1,3]$. Chest physicians often use available imagery (including chest radiographs or computed tomography scans) to identify the area of interest and determine the position of the patient $[1,2]$. The posterior chest is ideally scanned with the patient sitting using a bedside table as an armrest, whereas the lateral and anterior chest wall should be examined with the patient in either the lateral decubitus or supine position.

The pleura, peripheral lung tumours and consolidated lung can be visualised along the intercostal spaces [1]. Raising the arm above the patient's head increases the intercostal space distance and aids in scanning in erect or recumbent positions. Folding the patient's arms across their chest displaces the scapulae when scanning the upper posterior thorax. Visualisation of superior sulcus pathology can be achieved apically with the patient in the supine or sitting position.

Liberal application of gel is the final step prior to scanning [1]. It is advisable to hold the probe like a pen with the outer part of the hand in contact with the skin and to reduce ambient lighting. The sonographer should maintain visual contact with the screen while the dominant hand moves the probe across the area of interest and the other hand is used to optimise the digital image by adjusting the depth and gain. Findings can be compared with the contralateral side, which can be used as a control.

\section{The normal thorax}

The skin, muscles and facia planes are visible with a low-frequency probe as a series of echogenic layers [1-3]. Ribs appear as convex structures on transverse (vertical) scanning, with posterior acoustic shadowing. When viewed longitudinally, the anterior cortex appears as an uninterrupted echogenic line. The visceral and parietal pleura usually appear as a single highly echogenic line no more than $2 \mathrm{~mm}$ wide representing the pleura and pleuropulmonary interface [1-3]. On a longitudinal view, the pleural line will appear approximately $5 \mathrm{~mm}$ deep to the rib cortex [4]. The visceral and parietal pleura can be seen as two distinct echogenic lines on high-resolution scanning (fig. 1), with the latter seemingly thinner in appearance $[1,2]$. The two layers glide over each other during inspiration and expiration, which gives rise to the "lung sliding" sign on real-time ultrasonography, which is best appreciated on longitudinal (vertical) scanning [1-5]. Its presence has a high negative predictive value for the diagnosis of a pneumothorax [4-7].

Aerated lung parenchyma cannot be visualised on transthoracic ultrasonography $[1-3,8]$. The large change in acoustic impedance at the pleura-lung interface, however, results in horizontal artefacts, so called reverberation artefacts or A-lines, that are seen as a series of echogenic parallel lines equidistant from one another below the pleura [1-3, 7]. A few short, vertical "comet-tail" artefacts can be seen at the lung bases in normal individuals, and in all probability represent fluid-filled subpleural interlobular septa. B-lines are longer, pathological vertical artefacts that obliterate A-lines (see later) $[5,9]$.

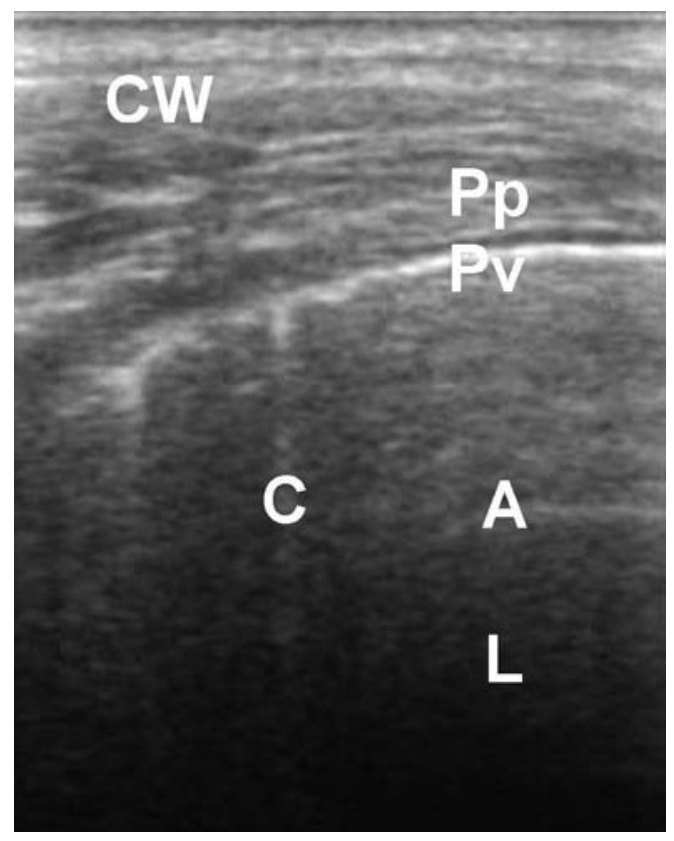

\section{Figure 1}

The high-frequency ultrasound appearance of a normal chest. The chest wall (CW) consists of multiple layers of echogenicity representing muscles and fascia. The more prominent visceral $(P v)$ and parietal pleura $(P p)$ are seen as echogenic bright lines that slide during respiration. A reverberation artefact, also known as an A-line (A), is present within the lung $(L)$, as well as a single comet tail artefact (C). 
The diaphragm is best visualised through the liver or the spleen $[1-3,5]$. It appears as an echogenic line, approximately $1-2 \mathrm{~mm}$ thick, which contracts with inspiration.

\section{Extrathoracic lymph nodes}

Ultrasonography provides visual access to cervical, supraclavicular and axillary lymph nodes, and it may aid in distinguishing malignant from reactive lymph nodes. Inflammatory lymph nodes have oval or triangular shapes and the fatty hilum appears echogenic on ultrasonography, whereas malignant nodes are often bulky and show loss of the fatty hilum leading to a hypoechoic appearance [1-3]. Irregular borders can be a sign of extracapsular spread. Ultrasonography has the added advantage that it can visualise supraclavicular or cervical lymph nodes in patients with superior vena cava obstruction, particularly when vascular congestion and swelling complicate physical palpation.

Supraclavicular lymph nodes are accessible to ultrasonography-guided fine-needle aspiration (FNA), which has the advantage of providing a cytological diagnosis and pathological staging $\left(\mathrm{pN}_{3}\right)$ in one procedure. FNA of extrathoracic lymph nodes can even be performed outside of a dedicated operating theatre at the patient's bedside [10].

\section{Chest wall pathology}

High-frequency ultrasound can detect softtissue masses arising from the chest wall, including lipomas, abscesses and many other (mostly benign) lesions. Masses generally have variable echogenicity and ultrasonographic findings are too nonspecific to differentiate between the various aetiologies [1-3]. Ultrasonography-assisted transthoracic FNA of these masses can readily be performed, as no aerated lung needs to be transversed during aspiration. Ultrasonography-assisted transthoracic FNA is generally performed under local anaesthesia, ideally with a 22-gauge injection-type or short spinal needle connected to a 10- $\mathrm{mL}$ syringe. The needle should contain a removable inner stylet to reduce contamination with chest wall tissue and blood $[1,3]$.

Rib fractures can also be visualised with ultrasound, which might even be more sensitive than radiography [11]. Bony metastases to the ribs can be detected by means of sonography and appear as hypoechoic masses that replace the normal echogenicity of the rib leading to a disruption of the cortical line [12]. Metastases are amenable to ultrasonography-assisted FNA.

Both chest wall masses and thoracic bony metastases can be sampled by means of cutting-needle biopsy (CNB). These devices are more invasive and carry a higher risk of visceral or vascular trauma [3, 13]. Potential visceral trauma can be caused by inadvertently passing the device through the ribs, as osteolytic rib metastases may provide less resistance than expected.

\section{Pleural pathology Pleural effusions}

Transthoracic ultrasonography is ideal for the detection and quantification of pleural effusions, and is more sensitive than decubitus expiratory films in identifying minimal or loculated effusions [14]. A pleural effusion is usually seen as a homogeneous, anechoic space between the parietal and visceral pleura (fig. 2). The space changes shape during respiration, although adhesions between the two pleural surfaces may result in the absence of lung motion above the effusion [1-3]. A tongue-like atelectatic lung is often seen inside a larger effusion.

Four ultrasonographic appearances of pleural effusions are recognised based on the internal echogenicity [1-3]:

1) anechoic

2) complex non-septated

3) complex septated

4) homogenously echogenic

Transudates typically appear as anechoic and non-septated, free-flowing effusions, whereas complex, septate or echogenic effusions are mostly exudates $[15,16]$. Malignant effusions are often anechoic, and pleural thickening $>10 \mathrm{~mm}$, pleural and diaphragmatic nodularity, and diaphragmatic thickening $>7 \mathrm{~mm}$ are highly suggestive of malignant disease [17]. Strands of echogenic material and septa that show more or less mobility with respiration are indicative of inflammatory effusions [1, 2]. The identification of septa (fig. 3) has clinical implications. Several studies have shown that patients with septated effusions needed longer chest tube drainage, longer hospital care and were more likely to require fibrinolytic therapy or surgery compared to those with non-septated effusions [18, 19]. 


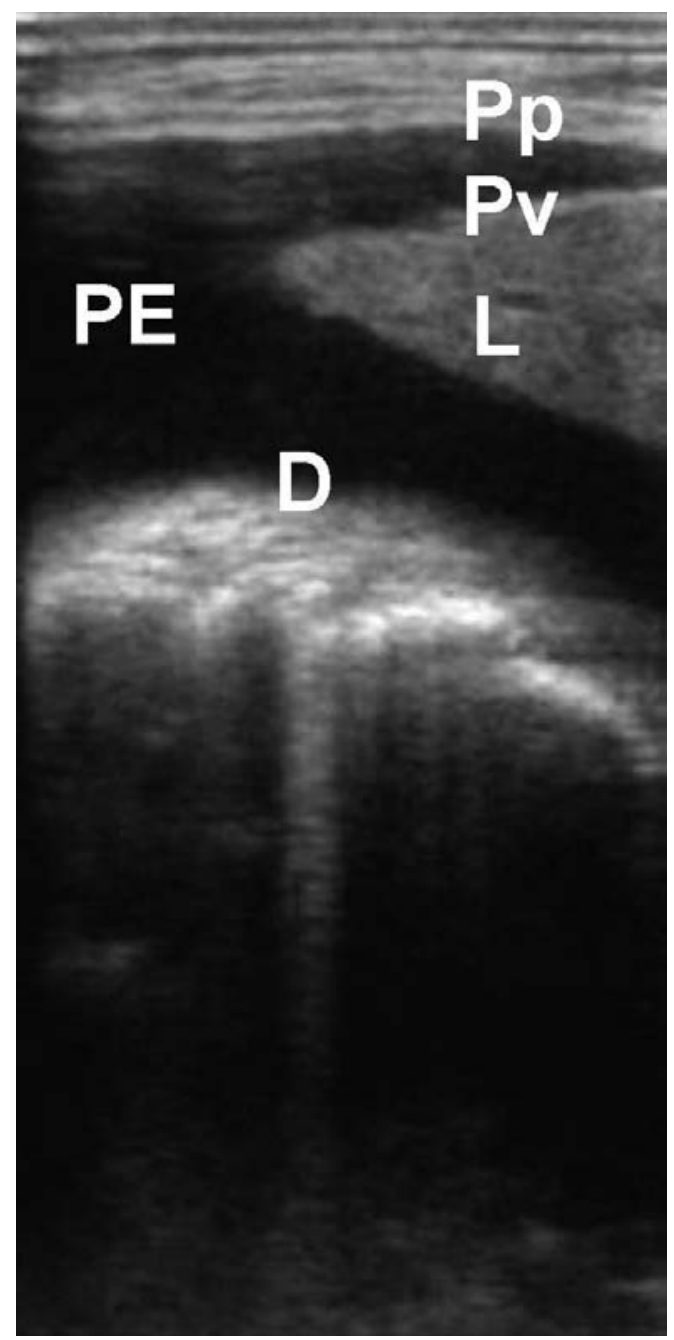

Figure 2

A pleural effusion (PE) gives rise to an anechoic space between the visceral $(P v)$ and parietal pleura $(P p)$.

Compressive atelectasis of the lung $(L)$ is seen on this high-frequency ultrasonogram. D: diaphragm.

One practical way to classify the volume of an effusion is simply to classify effusions as:

1) minimal, if the echo-free space is confined to the costophrenic angle

2) small, if the space is greater than the costophrenic angle but still within the range of the area covered with a $3.5-\mathrm{MHz}$ curvilinear probe

3) moderate, if the space is greater than a oneprobe range but within a two-probe range

4) large, if the space is greater than a twoprobe range [1-4]

Ultrasonographic guidance improves the success rate of pleural aspiration and minimises the risk of visceral puncture $[20,21]$. Moreover, the risk of pneumothorax following

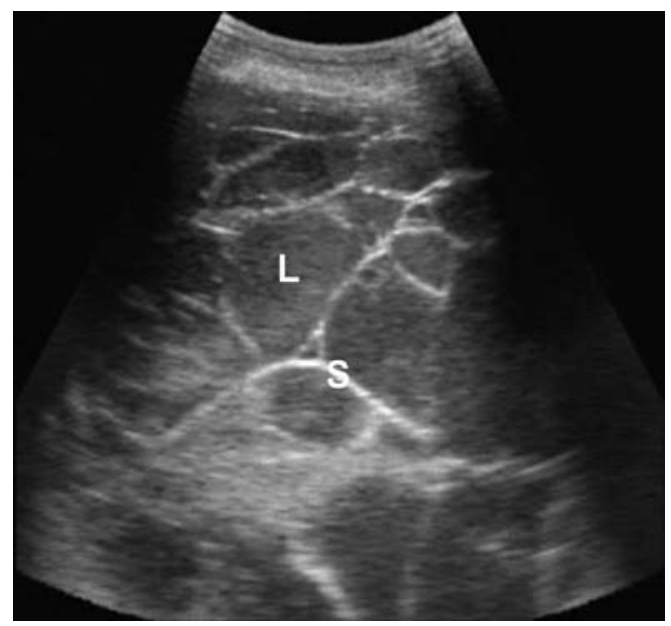

Figure 3

A complex septate effusion (viewed with lowfrequency ultrasonography) with multiple septa (S) and loculations ( $L$ ).

aspirations is reduced, irrespective of the size of the effusion [21]. Depending on operator experience, ultrasonography may also guide further decisions regarding the need for tube drainage, intrapleural fibrinolytic therapy, pleuroscopy or surgical intervention [22].

Ultrasonography is also ideal for identifying the optimal site for safe and effective intercostal drainage [21, 23]. Most operators utilise the "freehand" technique: a safe insertion site is identified and marked, then a drain is inserted. The intended site should have sufficient depth of pleural fluid of at least $10 \mathrm{~mm}$, no intervening lung and minimal risk of visceral trauma [23]. Ultrasonographic assistance does not always prevent laceration of the intercostal neurovascular bundle, as these structures may have a torturous course that may run medially to the angle of the rib $[23,24]$.

As an alternative, real-time ultrasonographic guidance may be employed. Ultrasonography is used for direct fluid aspiration, whereafter a guide wire is inserted, which is used to guide dilatation of a tract and deployment of a smallbore catheter (8-14 F). These tubes are better tolerated than large bore $(2 \mathrm{O}-24 \mathrm{~F})$ intercostal drains [25]. Some prospective studies have found that 8-12 $\mathrm{F}$ pigtail catheters or 10-14 $\mathrm{F}$ catheters inserted under ultrasonographic guidance are at least as effective as larger catheters [26-28].

Transthoracic ultrasonography is an extremely helpful guide for biopsies of the pleura $[1-3,12]$. Ultrasonography can identify focal pleural abnormalities. Moreover, measuring the 
size of an associated effusion decreases the risk of visceral lacerations, which is particularly important in cases with minimal pleural fluid [1, 3]. Unaided closed pleural biopsy has a very modest diagnostic yield of less than $60 \%$ for pleural malignancy [29]. The diagnostic yield of unaided closed pleural biopsy for pleural tuberculosis is generally much higher, due to the more homogenous distribution of tuberculous granulomata, and may even be as high as $87 \%$ if at least six specimens are harvested [30-32]. Recent studies have suggested that image-guidance may significantly increase the yield for malignancy while decreasing the risk of complications [12, 33-36]. CHANG et al. [35] found the diagnostic yield of ultrasonographyguided Tru-Cut (UK Medical Ltd, Sheffield, UK) pleural biopsy to be $77 \%$ for malignancies and DIACON et al. [36] showed that this figure may be as high as $100 \%$ for malignant mesothelioma extending at least $20 \mathrm{~mm}$ in any accessible dimension on ultrasonography. In a recent study, we found that the yield of an ultrasonographyassisted Abrams needle may be as high as $83 \%$ for malignant effusions [33].

\section{Pleural thickening}

Pleural thickening is often defined as a focal lesion that is greater than $3 \mathrm{~mm}$ in width, arising from the visceral or parietal pleura with or without an irregular margin [1-3]. Distinguishing pleural thickening from small effusions can be challenging, as both may appear hypoechoic on ultrasonography [37]. Lack of movement relative to the chest wall with respiration and the absence of a fluid colour sign seen with colour Doppler scanning are ultrasonographic evidence in favour of pleural thickening [1-3].

\section{Pneumothorax}

The diagnosis of a pneumothorax by means of ultrasonography requires more experience than the detection of pleural fluid [1-3]. Nevertheless, a recent meta-analysis concluded that bedside ultrasonography performed by clinicians had a higher sensitivity and similar specificity for the diagnosis of a pneumothorax when compared to chest radiography [38]. The absence of normal lung sliding and lung pulse, exaggerated horizontal reverberation artefacts (A-lines) and the absence of B-lines are reliable signs of a pneumothorax $[1,2,5,11,38]$. The "lung pulse" is caused by cardiac oscillation and refers to the subtle movements of the visceral pleura in relation to the parietal pleura [5]. In certain concomitant diseases, including diaphragmatic paralysis, prior pleurodesis or pleural adhesions and emphysema, the sensitivity of ultrasonography for the diagnosis of pneumothorax may be lower [1, 2, 38-40]. Ultrasonography may be used routinely to screen for post-procedural pneumothorax after transthoracic interventions as well as after transbronchial biopsy, and may obviate the need for routine post-procedural chest radiographs [41].

\section{Pulmonary pathology}

Practically any pathological process within the lung parenchyma can become detectable with ultrasonography, provided aerated lung tissue is replaced by consolidated or solid lung tissue and pleural contact is present. Often, the acoustic window is too narrow to allow assessment of the full dimension of the pathological process, but depth can almost always be determined accurately.

"Consolidation" is a nonspecific term referring to a subpleural, echo-poor region or one with tissue-like echotexture [5], which can be present in a range of diseases, including pneumonia, pulmonary embolism, lung tumours, atelectasis and pulmonary contusion. Additional signs may aid in distinguishing the various causes [5]. Pulmonary consolidation can easily be differentiated from an interstitial syndrome. Long, laser-like vertical hyperechoic artefacts that obliterate A-lines are called Blines, and are pathognomonic of an interstitial syndrome. Additional signs are needed to distinguishing the various causes, which may include pulmonary oedema and pulmonary fibrosis [5].

Early pneumonic consolidation appears diffusely echogenic and very similar in appearance to the texture of liver tissue. Both air and fluid bronchograms may be seen within the consolidated lung (fig. 4). Air bronchograms appear as dynamic echogenic branches and foci that fluctuate with the respiratory cycle $[1-3,5]$. Fluid bronchograms are seen as anechoic tubular structures and represent fluid-filled airways $[2,10]$. Furthermore, ultrasonography is ideally suited to screen for parapneumonic effusions and to differentiate dense consolidation from pleural effusions in critically ill patients (fig. 5). Ultrasonography-assisted transthoracic FNA of pneumonic consolidation is not often performed, yet it has a diagnostic yield greater 


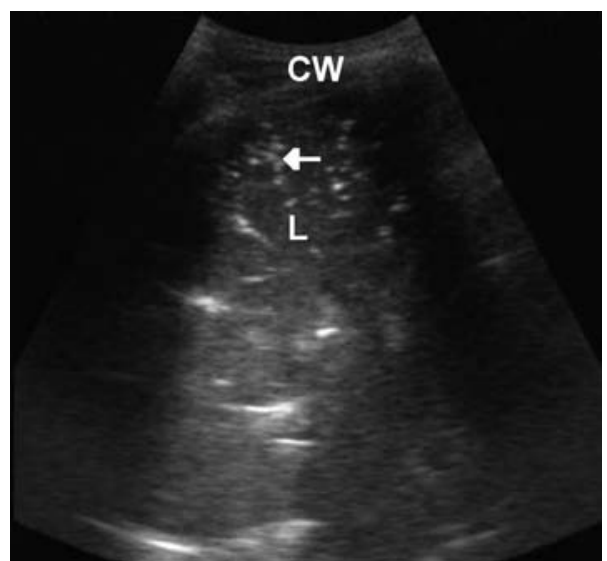

Figure 4

The low-frequency ultrasonographic appearance of a densely consolidated lung ( $L$ ) abutting the chest wall (CW). Note the multiple air bronchograms (echogenic branches and foci; arrow) and as well as fluid (hypodense) bronchograms.

than $90 \%$ with respect to the confirmation of the offending pathogen and has been shown to be safe [3]. Ultrasonography should also be used routinely to exclude a pneumothorax following transthoracic FNA, particularly in mechanically ventilated patients.

Lung abscesses abutting the chest wall pleura appear as hypoechoic lesions with a well-defined or irregular wall [1-3]. The centre of the abscess is most often anechoic, but internal echoes may be seen. It is worthwhile scanning the patient in different positions if an air-fluid level is suspected, as the appearance

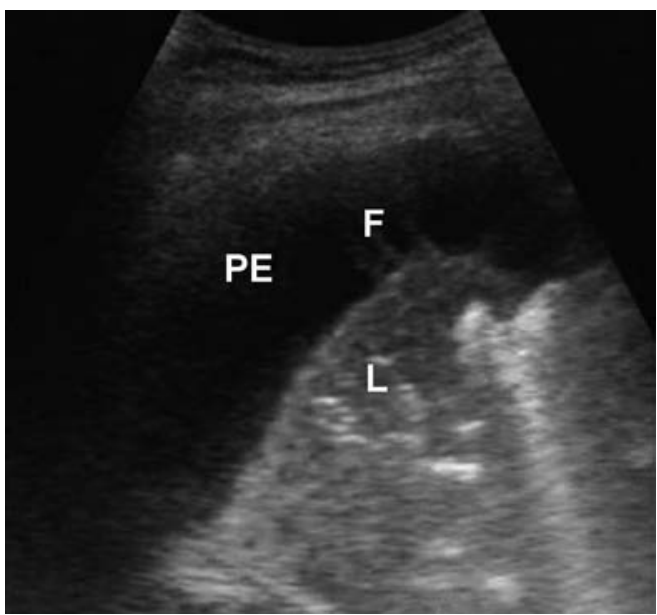

Figure 5

This low-frequency ultrasonogram confirmed the presence of a parapneumonic pleural effusion (PE). Note the early fibrin strands (F) extending from the visceral pleura of the underlying consolidated lung ( $L$ ). may alter accordingly. The vast majority of lung abscesses are visible on ultrasonography and transthoracic FNA is more than 90\% likely to be diagnostic for a specific pathogen.

A hypoechoic appearance with posterior acoustic enhancement and irregular borders is typical for lung tumours (fig. 6). The visceral pleural line is interrupted once the visceral pleura is infiltrated, and loss of movement with respiration on low- or high-frequency scanning is indicative of extension beyond the parietal pleura. In fact, high-resolution ultrasonographic scanning is known to be superior to routine chest computed tomography scanning in evaluating tumour invasion of the pleura and chest wall [42, 43], which has a direct impact on tumour staging.

For ultrasonography-assisted biopsy of pulmonary tumours, the technique is used by most clinicians. It can be utilised for both ultrasonography-assisted transthoracic FNA and CNB. Following patient positioning, the intended site of needle insertion is identified by ultrasonography and marked while the direction, depth of interest and safety range for the procedure are determined $[1,2]$. It is essential that the patient must not alter their position in order to prevent a positional shift of the area of interest relative to the skin mark. Transthoracic FNA can be performed with spinal needles of various sizes (usually 22-gauge) and lengths (40 or $90 \mathrm{~mm}$ ) connected to a $10-\mathrm{mL}$ syringe, with

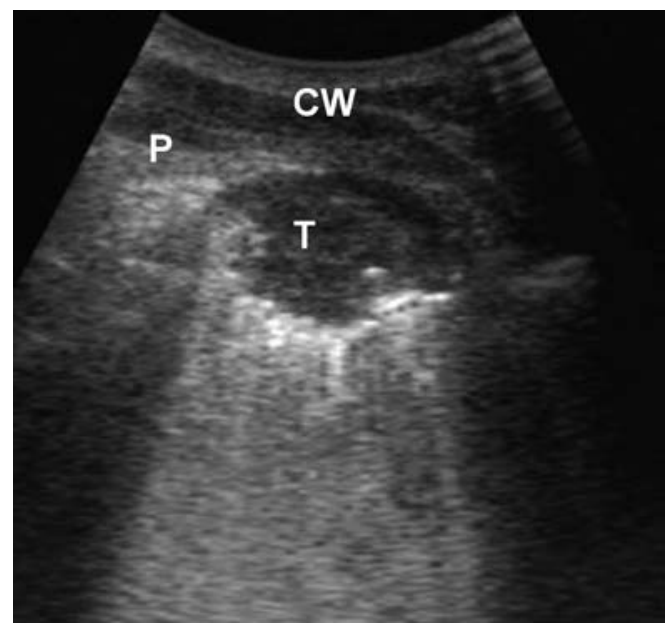

Figure 6

A low-frequency ultrasonographic image of a peripheral lung tumour infiltrating the chest wall. Note that the acoustic window is too narrow to demonstrate the whole circumference of the lesion, but it allows determination of its full depth. Note the extension of the lung tumour $(T)$ beyond the pleura $(P)$ into the chest wall (CW). 
local anaesthesia (lignocaine, 1\%). Aspirates from at least four slightly different directions and depths should be collected by moving the needle in and out while applying a negative pressure. The material should immediately be expressed onto slides and stained. Rapid on-site evaluation (ROSE) of the cytology specimens, if available, should be employed to assess the need for CNB in the same setting. CNB will harvest tissue but carries a higher risk of visceral injury and pneumothorax. It should only be used if a sufficient safety range can be assured. Current evidence suggest that ultrasonographyassisted transthoracic FNA is superior to CNB in confirming a diagnosis of epitheloid carcinomas of the lung ( $95 \%$ versus $81 \%$ ), whereas CNB is superior in cases of benign lesions and noncarcinomatous tumours $[44,45]$.

Pulmonary embolism may give rise to infarction, which is visualised on ultrasonography as a peripheral wedge-shaped hypoechoic region, with or without an associated pleural effusion $[46,47]$. Pulmonary embolism should be considered to be present when more than one characteristic triangular pleura-based lesion is present and probable if at least one typical lesion with a corresponding pleural effusion is present [47]. MATHIS et al. [47] found these criteria to have a sensitivity of $74 \%$, specificity of $95 \%$, positive predictive value of $95 \%$ and negative predictive of $75 \%$ for pulmonary embolism.

B-lines generally extend to the bottom of the screen and are considered pathological and indicative of an interstitial syndrome [5]. "Lung rockets" refer to the presence of several B-lines [39]. LICHTENSTEIN et al. [48] found that B-lines were absent in most patients with chronic obstructive airway disease but present in more than $90 \%$ of patients with interstitial syndromes. The presence of bilateral B-lines in the setting of patients with acute dyspnoea is, therefore, a very reliable sign to differentiate patients with pulmonary oedema (fig. 7) from those with chronic obstructive airway disease $[5,48]$. Acute respiratory distress syndrome and pulmonary fibrosis tend to give rise to more patchy involvement (with spared areas) and a nonhomogeneous distribution of Blines, as well as an irregular thickened or fragmented pleural line [5].

Another indication for the use of transthoracic ultrasonography is the assessment of pulmonary and pleural-based cysts including, for example, hydatid disease (fig. 8). Cysts commonly appear as large, round anechoic lesions. Rounded atelectasis may give rise to a pleural based mass with associated pleural thickening and extrapleural fat. The invaginated pleura may be visualised as an echogenic line running from the pleura into the apparent mass.

\section{Mediastinal pathology}

The differential diagnosis of mediastinal masses is broad and computed tomography scanning followed by biopsy is performed in practically all cases. Mediastinoscopy, mediastinotomy or related surgical procedures have diagnostic yields in excess of $90 \%$, but they have to be performed in theatre under general anaesthesia and are associated with a complication rate of up to $5 \%$.

Ultrasonography-assisted biopsy of mediastinal masses provides a minimally invasive, safe and cheap alternative to mediastinoscopy or mediastinotomy in the assessment of mediastinal masses [7]. Attention needs to be paid to identifying a safe biopsy tract, given

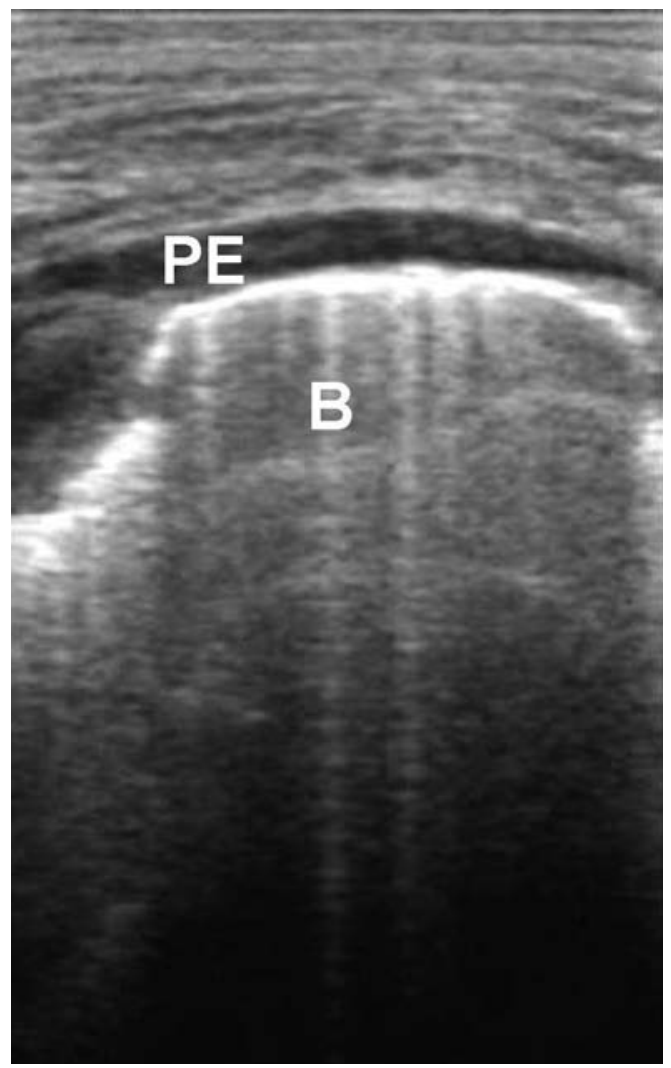

Figure 7

This high-frequency ultrasonogram was obtained from a patient with severe mitral stenosis who presented with pulmonary oedema. Note the widespread B-lines (B), a reliable sign of interstitial pulmonary oedema, as well as the associated small pleural effusion (PE). 


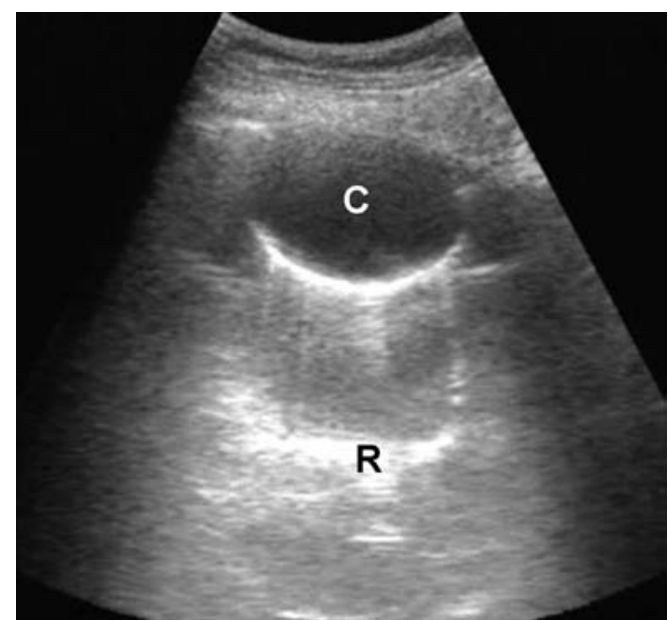

Figure 8

The low-frequency ultrasound appearance of a hydatid cyst. A reverberation artefact $(R)$ is present due to the high water content of the cyst (C).

the vicinity of the heart and great vessels. Furthermore, mediastinal structures may be significantly displaced in the diseased state.

In a recent study we performed ultrasonography-assisted transthoracic FNA with ROSE on patients with anterior mediastinal masses, immediately followed by CNB in cases where a provisional diagnosis of epithelial carcinoma or tuberculosis could not be established [49]. An accurate cytological diagnosis was made in $73 \%$ of cases, and was more likely to be positive in cases of epithelial carcinoma or tuberculosis than all other pathology $(p<0.001)$. CNB yielded a diagnosis in $88 \%$. Overall, $93 \%$ of patients were diagnosed by this sequential single-session approach without the assistance of a specialist radiologist or thoracic surgeon.

\section{Conclusions}

All trainees in thoracic medicine should be taught the basics transthoracic ultrasonography, given its usefulness as a diagnostic modality, and its ability to guide diagnostic and therapeutic interventions. The investigation of chest wall abnormalities, pleural thickening and pleural tumours, and the qualitative and quantitative description of pleural effusions remain the major indications for the use of transthoracic ultrasonography. Other indications include the visualisation of lung tumours, pulmonary consolidation and other parenchymal pulmonary processes provided they abut the pleura. Ultrasonography is the ideal tool to assist with pleural aspiration. Ultrasonography-assisted biopsy of lesions arising from the chest wall, pleura, peripheral lung and mediastinum is safe and has a high yield comparable to more invasive techniques. Other applications of transthoracic ultrasonography include the diagnosis of a pneumothorax and pulmonary embolism.

Tips for clinical practice

- Take sufficient time to appropriately position the patient. Use available imagery to be guided in terms of area of interest and patient position.

- Appreciate that peripheral lymph nodes, chest wall pathology, anterior mediastinal masses and consolidating pulmonary pathology that abut the chest wall can be visualised by means of transthoracic ultrasonography.

- Classify a pleural effusion as minimal, moderate or large based on ultrasonographic assessment.

- Look for the absence of normal lung sliding and lung pulse, exaggerated horizontal reverberation artefacts (A-lines), the absence of B-lines and the presence of a lung point to diagnose a pneumothorax.

- Use the freehand technique to perform biopsy of various pathologies. Make sure the patient does not alter their position during the procedure and use adequate local analgesia (to minimise pain and to avoid sudden movements).

Furthermore, avoid puncture of intercostal arteries (inferior to the ribs), the internal thoracic/mammarian artery $(1-2 \mathrm{~cm}$ lateral to the sternum), and subclavian and major intrathoracic vessels when attempting a biopsy.

- Always identify the diaphragm and subdiaphragmatic viscera prior to performing lower transthoracic biopsies, as these structures may be displaced cephalad, given a false impression of a solid mass or consolidated lung.

- Once a specimen is obtained by means of needle aspiration for cytology, expel material onto slides within seconds. The quality of smear preparation is as important as the biopsy technique.

- Utilise ROSE to provisionally confirm the presence of diagnostic material.

- Where epithelial carcinoma or an infectious process are not suspected or identified on ROSE, perform a CNB, provided a safety range can be guaranteed.

- Use ultrasonography to screen for post-procedural pneumothoraces. 


\section{References}

1. Koegelenberg CFN, Bolliger CT, Diacon AH. Pleural Ultrasound, In: Light RW, Lee YC. Textbook of Pleural Disease. 2nd Edn. London, Hodder \& Stoughton, 2008; pp. 275-283.

2. Koh DM, Burke S, Davies N, et al. Transthoracic US of the chest: clinical uses and applications. Radiographics 2002; 22: E1.

3. Koegelenberg CFN, Diacon AH, Bolliger CT. Transthoracic ultrasound of the chest wall, pleura, and the peripheral lung, In: Bollger CT, Herth FJF, Mayo $\mathrm{PH}$, et al. Progress in Respiratory Research. Clinical Chest Ultrasound. Basel, Karger, 2009; vol. 37: pp. 22-33.

4. Koenig SJ, Narasimhan M, Mayo PH. Thoracic ultrasonography for the pulmonary specialist. Chest 2011; 140: 1332-1341.

5. Volpicelli G, Elbarbary M, Blaivas $M$, et al. International evidence-based recommendations for point-of-care lung ultrasound. Intensive Care Med 2012; 38: 577-591.

6. Tsai TH, Jemg J-S, Yang P-C. Clinical applications of transthoracic ultrasound in chest medicine. J Med Ultrasound 2008; 16: 7-25.

7. Lichtenstein DA, Menu Y. A bedside ultrasound sign ruling out pneumothorax in the critically ill. Chest 1995; 108: 1345-1348.

8. Tsai TH, Yang PC. Ultrasound in the diagnosis and management of pleural disease. Curr Opin Pulm Med 2003; 9: 282-290.

9. Lichtenstein DA, Mezière GA. Relevance of lung ultrasound in the diagnosis of acute respiratory failure: the BLUE protocol. Chest 2008; 134: 117-125.

10. Hoosen MM, Barnes D, Khan AN, et al. The importance of ultrasound in staging and gaining a pathological diagnosis in patients with lung cancer a two year single centre experience. Thorax 2011; 66: 414-417.

11. Mathis G. Thoraxsonography. I. Chest and pleura. Ultrasound Med Biol 1997; 23: 1131-1139.

12. Bitschnau R, Gehmacher O, Kopf A, et al. Ultrasound in the diagnosis of rib and sternal fracture. Ultraschall Med 1997; 18: 158-161.

13. Koegelenberg CF, Diacon AH. Pleural controversy: closed needle pleural biopsy or thoracoscopy - which first? Respirology 2011; 16: 738-746.

14. Kocijancic I, Kocijancic K, Cufer T. Imaging of pleural fluid in healthy individuals. Clin Radiol 2004; 59: 826-829.

15. Yang PC, Luh KT, Chang DB. Value of sonography in determining the nature of pleural effusion: analysis of 320 cases. AJR Am J Roentgenol 1992; 159: 29-33.

16. Hirsch JH, Rogers JV, Mack LA. Real-time sonography of pleural opacities. Am J Roentgenol 1981; 136: 297-301.

17. Qureshi NR, Rahman NM, Gleeson FV. Thoracic ultrasound in the diagnosis of malignant pleural effusion. Thorax 2009; 64: 139-143.

18. Chen KY, Liaw YS, Wang HC, et al. Sonographic septation: a useful prognostic indicator of acute thoracic empyema. J Ultrasound Med 2000; 19: 837-843.

19. Tu CY, Hsu WH, Hsia TC, et al. Pleural effusions in febrile medical ICU patients: chest ultrasound study. Chest 2004; 126: 1274-1280.

20. Diacon AH, Brutsche MH, Solèr M. Accuracy of pleural puncture sites: a prospective comparison of clinical examination with ultrasound. Chest 2003; 123 : 436-441.
21. Hooper C, Lee YC, Maskell N. Investigation of a unilateral pleural effusion in adults: British Thoracic Society pleural disease guideline 2010. Thorax 2010; 65: Suppl. 2, ii4-ii17.

22. Chen KY, Liaw YS, Wang HC. Sonographic septation: a useful prognostic indicator of acute thoracic empyema. J Ultrasound Med 2000; 19: 837-843.

23. Havelock T, Teoh R, Laws D, et al. Pleural procedures and thoracic ultrasound: British Thoracic Society pleural disease guideline 2010. Thorax 2010; 65: Suppl. 2, ii61-ii76.

24. Doelken P, Strange C. Chest ultrasound for "dummies". Chest 2003; 123: 332-333.

25. Tatersall DJ, Traill ZC, Gleeson FV. Chest drains: does size matter? Clin Radiol 2000; 55: 415-421.

26. Shankar S, Gulati M, Kang M, et al. Image-guided percutaneous drainage of thoracic empyema: can sonography predict the outcome? Eur Radiol 2000; 10: 495-499.

27. Ali I, Unruh H. Management of empyema thoracis. Ann Thorac Surg 1990; 50: 355-359.

28. Keeling AN, Leong S, Logan PM, et al. Empyema and effusion: outcome of image-guided small-bore catheter drainage. Cardiovasc Intervent Radiol 2008; 31: 135-141.

29. Tomlinson JR. Invasive procedures in the diagnosis of pleural disease. Semin Respir Med 1987; 9: 30-60.

30. Valdés L, Alvarez D, San José E, et al. Tuberculous pleurisy: a study of 254 patients. Arch Intern Med 1998; 158: 2017-2021.

31. Diacon AH, Van de Wal BW, Wyser C, et al. Diagnostic tools in tuberculous pleurisy: a direct comparative study. Eur Respir J 2003; 22: 589-591.

32. Kirsch CM, Kroe DM, Azzi RL, et al. The optimal number of pleural biopsy specimens for a diagnosis of tuberculous pleurisy. Chest 1997; 112: 702-706.

33. Koegelenberg CF, Bolliger CT, Theron J, et al. Direct comparison of the diagnostic yield of ultrasoundassisted Abrams and Tru-Cut needle biopsies for pleural tuberculosis. Thorax 2010; 65: 857-862.

34. Metintas M, Ak G, Dundar E, et al. Medical thoracoscopy vs CT scan-guided Abrams pleural needle biopsy for diagnosis of patients with pleural effusions: a randomized, controlled trial. Chest 2010; 137: 1362-1368.

35. Chang BD, Yang PC, Luh KT, et al. Ultrasound-guided pleural biopsy with Tru-Cut needle. Chest 1991; 100: 1328-1333.

36. Diacon AH, Schuurmans MM, Theron J, et al. Safety and yield of ultrasound assisted transthoracic biopsy performed by pulmonologists. Respiration 2004; 71: 519-522.

37. Marks WM, Filly RA, Callen PW. Real-time evaluation of pleural lesions: new observations regarding the probability of obtaining free fluid. Radiology 1982; 142: $163-164$.

38. Ding W, Shen $Y$, Yang J, et al. Diagnosis of pneumothorax by radiography and ultrasonography: a meta-analysis. Chest 2011; 140: 859-866.

39. Gottesman E, McCool MD. Ultrasound evaluation of the paralyzed diaphragm. Am J Resp Crit Care Med 1997; 155: 1570-1574.

40. Chan SS. The comet tail artefact in the diagnosis of pneumothorax. J Ultrasound Med 2002; 21: 1060.

41. Kreuter M, Eberhardt $\mathrm{R}, \mathrm{Wenz} \mathrm{H}$, et al. The correct one is: diagnostic value of transthoracic ultrasound compared to chest radiography in the detection of a post-interventional pneumothorax. Ultraschall Med 2O11; 32: E2O-E23. 
42. Sugama $\mathrm{Y}$, Tamaki $\mathrm{S}$, Kitamura $\mathrm{S}$, et al. Ultrasonographic evaluation of pleural and chest wall invasion of lung cancer. Chest 1988; 93: 275-279.

43. Suzuki N, Saitoh T, Kitamura S. Tumor invasion of the chest wall in lung cancer: diagnosis with US. Radiology 1993; 187: 39-42.

44. Diacon AH, Theron J, Schubert P, et al. Ultrasoundassisted transthoracic biopsy: fine-needle aspiration or cutting-needle biopsy? Eur Respir J 2007; 29: 357-362.

45. Schubert P, Wright CA, Louw M, et al. Ultrasoundassisted transthoracic biopsy: cells or section? Diagn Cytopathol 2005; 33: 233-237.

46. Mathis G, Metzler J, Fubenegger D. Sonographic observation of pulmonary infarction and early infarctions by pulmonary embolism. Eur Heart J 1993; 14: 804-808.

47. Mathis G, Blank W, Reissig A, et al. Thoracic ultrasound for diagnosing pulmonary embolism: a prospective multicenter study of 352 patients. Chest 2005; 128: 1531-1538.

48. Lichtenstein D, Mézière $G$, Biderman $P$, et al. The comet-tail artefact: an ultrasound sign of alveolarinterstitial syndrome. Am J Respir Crit Care Med 1997; 156: $1640-1646$.

49. Koegelenberg CF, Bolliger CT, Irusen EM, et al. The diagnostic yield and safety of ultrasound-assisted biopsy of mediastinal masses. Respiration 2011; 81: 134-141. 